\title{
Distances, Hesitancy Degree and Flexible Querying via Neutrosophic Sets
}

\author{
A.A.Salama \\ Math and Computer Science \\ Department, Faculty of Science \\ Port Said University, EGYPT
}

\author{
Mohamed Abdelfattah \\ Information System \\ Department, Faculty of \\ Computers \& Information, \\ Benha University,
}

\author{
Mohamed Eisa \\ Computer Science Department, \\ Port Said University, \\ Egypt
}

\begin{abstract}
Since the world is full of indeterminacy, the neutrosophics found their place into contemporary research. İn this paper we, introduce the distances between neutrosophic sets: the Hamming distance, The normalized Hamming distance, the Euclidean distance and normalized Euclidean distance. We will extend the concepts of distances to the case of neutrosophic hesitancy degree. Added to, this paper suggest how to enrich intuitionistic fuzzy querying by the use of neutrosophic values..
\end{abstract}

\section{General Terms}

Your general terms must be any term which can be used for general classification of the submitted material such as Pattern Recognition, Security, Algorithms et. al.

\section{Keywords}

Neutrosophic Sets; Hamming distance; Euclidean Distance; Normalized Euclidean Distance; Intuitionistic Fuzzy Querying; Querying Databases; Neutrosophic Querying.

\section{INTRODUCTION}

Since the world is full of indeterminacy, the neutrosophics found their place into contemporary research. The fundamental concepts of neutrosophic set, introduced by Smarandache in $[15,16]$, and Salama et al. in $[4,10,11,12$, $13,14,18,19,20,21,22,23]$, provides a natural foundation for treating mathematically the neutrosophic phenomena which exist pervasively in our real world and for building new branches of neutrosophic mathematics. Neutrosophy has laid the foundation for a whole family of new mathematical theories generalizing both their classical and fuzzy counterparts $[1,2,3,5,6,7,8,17,24,25]$ such as a neutrosophic set theory.The traditional query languages, used in the database management systems, require a precise and unambiguous specification of a query. It seems to be a serious limitation since a typical user often formulates his requirements in a natural language using imprecise expressions and vague terms. For this reason several approaches have been proposed to relax the rigidity of the conventional queries and make possible to use queries that allow for a more intelligent and human consistent information retrieval (see, e.g. $[5,8,9]$ ). The FQUERY for Access in [5, 6, $7,8,9]$ is an example of a computer program that enables to create different kinds of fuzzy queries. Using such fuzzy queries we deal no longer with binary outputs whether a record fulfill given requirement or not - but we get an Definition 3.1 information on the degree the record complies with the requirement. İn this paper we, introduce the distances between neutrosophic sets: the Hamming distance, The normalized Hamming distance, the Euclidean distance and normalized Euclidean distance. We will extend the concepts of distances to the case of neutrosophic hesitancy degree.

\section{TERMINOLOGIES}

Neutrosophy has laid the foundation for a whole family of new mathematical theories generalizing both their classical and fuzzy counterparts $[1,2,3,5,6,7,8,17,24,25]$ such as a neutrosophic set theory. We recollect some relevant basic preliminaries, and in particular, the work of Smarandache in $[15,16]$ and Salama et al. $[4,10,11,12,13,14,18,19,20$, 21, 22, 23]. Smarandache introduced the neutrosophic components T, I, F which represent the membership, indeterminacy, and non-membership values respectively, where is nonstandard unit interval. Salama introduced the following: Let $\mathrm{X}$ be a non-empty fixed set. A neutrosophic set is an object having the form where and which represent the degree of member ship function (namely ), the degree of indeterminacy (namely), and the degree of non-member ship (namely ) respectively of each element to the set where and . Smarandache introduced the following: Let T, I, F be real standard or nonstandard subsets of , with

Sup_T=t_sup, inf_T=t_inf

Sup_I=i_sup, inf_I=i_inf

Sup_F=f_sup, inf_F=f_inf

n-sup=t_sup + i_sup + f_sup

n-inf $=\mathrm{t} \_$inf $+\mathrm{i} \_$inf $+\mathrm{f} \_$inf,

T, I, F are called neutrosophic components

\section{DISTANCES BETWEEN NEUTROSOPHIC SETS}

We will now extend the concepts of distances presented in [17] to the case of neutrosophic sets. 

then

et $A=\left\{\left(\mu_{A}(x), v_{A}(x), \gamma_{A}(x)\right), x \in X\right\}$ and $B=\left\{\left(\mu_{B}(x), v_{B}(x), \gamma_{B}(x)\right), x \in X\right\}$ in $X=\left\{x_{1}, x_{2}, x_{3}, \ldots, x_{n}\right\}$

$$
d_{N s}(A, B)=\sum_{i=1}^{n}\left(\left|\mu_{A}\left(x_{i}\right)-\mu_{B}\left(x_{i}\right)\right|+\left|v_{A}\left(x_{i}\right)-v_{B}\left(x_{i}\right)\right|+\left|\gamma_{A}\left(x_{i}\right)-\gamma_{B}\left(x_{i}\right)\right|\right) \text {. }
$$

ii) The

$$
e_{N s}(A, B)=\sqrt{\sum_{i=1}^{n}\left(\left(\mu_{A}\left(x_{i}\right)-\mu_{B}\left(x_{i}\right)\right)^{2}+\left(v_{A}\left(x_{i}\right)-v_{B}\left(x_{i}\right)\right)^{2}+\left(\gamma_{A}\left(x_{i}\right)-\gamma_{B}\left(x_{i}\right)\right)^{2}\right)}
$$

iii) The

$$
N H_{N s}(A, B)=\frac{1}{2 n} \sum_{i=1}^{n}\left(\left|\mu_{A}\left(x_{i}\right)-\mu_{B}\left(x_{i}\right)\right|+\left|v_{A}\left(x_{i}\right)-v_{B}\left(x_{i}\right)\right|+\left|\gamma_{A}\left(x_{i}\right)-\gamma_{B}\left(x_{i}\right)\right|\right)
$$

iv) The

Euclidean distance is equal

$$
N E_{N S}(A, B)=\sqrt{\frac{1}{2 n} \sum_{i=1}^{n}\left(\left(\mu_{A}\left(x_{i}\right)-\mu_{B}\left(x_{i}\right)\right)^{2}+\left(v_{A}\left(x_{i}\right)-v_{B}\left(x_{i}\right)\right)^{2}+\left(\gamma_{A}\left(x_{i}\right)-\gamma_{B}\left(x_{i}\right)\right)^{2}\right)}
$$

\section{Example 3.1}

Let us consider for simplicity degenrated neutrosophic sets $A, B, D, G, F$ in $X=\{a\}$. A full description of each neutrosophic set i.e. $A=\left\{\left(\mu_{A}(x), v_{A}(x), \gamma_{A}(x)\right), a \in X\right\}$, may be exemplified by $A=\{\langle 1,0,0\rangle, a \in X\}, B=\{\langle 0,1,0\rangle, a \in X\}$,

$$
\begin{aligned}
& D=\{\langle 0,0,1\rangle, a \in X\}, G=\{\langle 0.5,0.5,0\rangle, a \in X\}, \\
& E=\{\langle 0.25,0.25,0.0 .5\rangle, a \in X\}, \quad \text { Let us calculate four }
\end{aligned}
$$
distances between the above neutrosophic sets using i), ii), iii) and iv) formulas ,

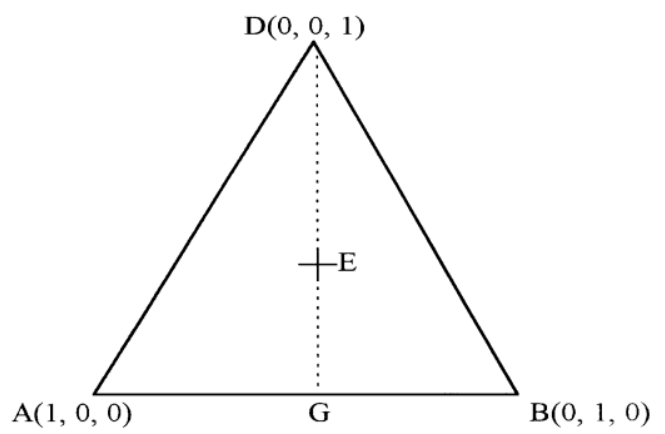

Fig.1A geometrical interpretation of the neutrosophic considered in Example 5.1 .

We obtain $e_{N S}(A, D)=\frac{1}{2}, e_{N s}(B, D)=\frac{1}{2}, e_{N s}(A, B)=\frac{1}{2}, e_{N s}(A, G)=\frac{1}{2}, e_{N S}(B, G)=\frac{1}{2}, e_{N s}(E, G)=\frac{1}{4}, e_{N s}(D, G)=\frac{1}{4}$, $N E_{N s}(A, B)=1, N E_{N S}(A, D)=1, N E_{N s}(B, D)=1, N E_{N s}(A, G)=\frac{1}{2}, N E_{N s}(B, G)=\frac{1}{2}, N E_{N s}(B, G)=\frac{1}{2}$,

$N E_{N S}(E, G)=\frac{\sqrt{3}}{4}$, and $N E_{N s}(D, G)=\frac{\sqrt{3}}{2}$,

From the above results the triangle ABD (Fig.1) has edges equal to $\sqrt{2}$ and $e_{N s}(A, D)=e_{N s}(B, D)=e_{N s}(A, B)=\frac{1}{2}$ and $N E_{N s}(A, B)=N E_{N s}(A, D)=N E_{N s}(B, D)=$ $2 N E_{N S}(A, G)=2 N E_{N S}(B, G)=1$, and $\quad N E_{N S}(E, G)$ is equal to half of the height of triangle with all edges equal to $\sqrt{2}$ multiplied by, $\frac{1}{\sqrt{2}}$ i.e. $\frac{\sqrt{3}}{4}$.

Then $d_{N S}(A, B)=3, N H_{N S}(A, B)=0.43, e_{N S}(A, B)=1.49$ and $N E_{N S}(A, B)=0.55$.

\section{Remark 3.1}

Clearly these distances satisfy the conditions of metric space. Remark 3.2

\section{Example 3.2}

Let us consider the following neutrosophic sets $\mathrm{A}$ and $\mathrm{B}$ in $X=\{a, b, c, d, e\}$,

$A=\{\langle 0.5,0.3,0.2\rangle,\langle 0.2,0.6,0.2\rangle,\langle 0.3,0.2,0.5\rangle,\langle 0.2,0.2,0.6\rangle,\langle 1,0,0\rangle\}$

$B=\{\langle 0.2,0.6,0.2\rangle,\langle 0.3,0.2,0.5\rangle,\langle 0.5,0.2,0.3\rangle,\langle 0.9,0,0.1\rangle,\langle 0,0,0\rangle\}$
It is easy to notice that for formulas i), ii), iii) and iv) the following is valid: 

a) $0 \leq d_{N s}(A, B) \leq n$
b) $0 \leq N H_{N s}(A, B) \leq 1$
c) $0 \leq e_{N s}(A, B) \leq \sqrt{n}$

d) $0 \leq N E_{N s}(A, B) \leq 1$.

This representation of a neutrosophic set (Fig. 2) will be a point of departure for neutrosophic crisp distances, and entropy of neutrosophic sets.

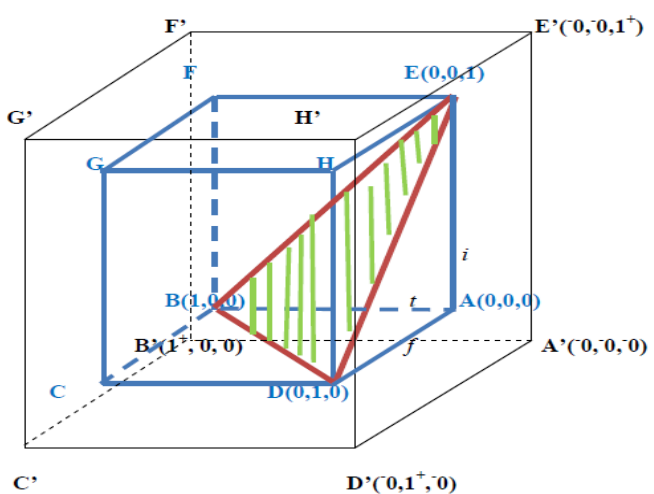

Fig. 2. A three-dimension representation of a neutrosophic set.

We will now extend the concepts of distances to the case of neutrosophic hesitancy degree. By taking into account the four parameters characterization of neutrosophic sets i.e. $A=\left\{<\mu_{A}(x), v_{A}(x), \gamma_{A}(x), \pi_{A}(x)>, x \in X\right\}$

\section{Definition3.2}

Let $A=\left\{\left(\mu_{A}(x), v_{A}(x), \gamma_{A}(x)\right), x \in X\right\}$ and $B=\left\{\left(\mu_{B}(x), v_{B}(x), \gamma_{B}(x)\right), x \in X\right\}$ on $X=\left\{x_{1}, x_{2}, x_{3}, \ldots, x_{n}\right\}$ For a neutrosophic set $A=\left\{\left(\mu_{A}(x), v_{A}(x), \gamma_{A}(x)\right), x \in X\right\}$ in X, we call $\pi_{A}(x)=3-\mu_{A}(x)-v_{A}(x)-\gamma_{A}(x)$, the neutrosophic index of $\mathrm{x}$ in $\mathrm{A}$. İt is a hesitancy degree of $\mathrm{x}$ to $\mathrm{A}$ it is obvtous that $0 \leq \pi_{A}(x) \leq 3$.

\section{Definition 3.3}

$$
\text { Let } \quad A=\left\{\left(\mu_{A}(x), v_{A}(x), \gamma_{A}(x)\right), x \in X\right\} \quad \text { and } B=\left\{\left(\mu_{B}(x), v_{B}(x), \gamma_{B}(x)\right), x \in X\right\} \text { in }
$$
$X=\left\{x_{1}, x_{2}, x_{3}, \ldots, x_{n}\right\}$ then

i) The Hamming distance is equal to

ii) $\quad d_{N s}(A, B)=\sum_{i=1}^{n}\left(\left|\mu_{A}\left(x_{i}\right)-\mu_{B}\left(x_{i}\right)\right|+\left|v_{A}\left(x_{i}\right)-v_{B}\left(x_{i}\right)\right|+\left|\gamma_{A}\left(x_{i}\right)-\gamma_{B}\left(x_{i}\right)\right|+\left|\pi_{A}\left(x_{i}\right)-\pi_{B}\left(x_{i}\right)\right|\right)$

iii) .Taking into account that

$\pi_{A}\left(x_{i}\right)=3-\mu_{A}\left(x_{i}\right)-v_{A}\left(x_{i}\right)-\gamma_{A}\left(x_{i}\right)$ and $\pi_{B}\left(x_{i}\right)=3-\mu_{B}\left(x_{i}\right)-v_{B}\left(x_{i}\right)-\gamma_{B}\left(x_{i}\right)$

we have

$\left|\pi_{A}\left(x_{i}\right)-\pi_{B}\left(x_{i}\right)\right|=\left|3-\mu_{A}\left(x_{i}\right)-v_{A}\left(x_{i}\right)-\gamma_{A}\left(x_{i}\right)-3+\mu_{A}\left(x_{i}\right)+v_{B}\left(x_{i}\right)+\gamma_{B}\left(x_{i}\right)\right|$

$\leq\left|\mu_{B}\left(x_{i}\right)-\mu_{A}\left(x_{i}\right)\right|+\left|v_{B}\left(x_{i}\right)-v_{A}\left(x_{i}\right)\right|+\left|\gamma_{B}\left(x_{i}\right)-\gamma_{A}\left(x_{i}\right)\right|$.

iv) The

Euclidean

distance

is

equal

to

$$
e_{N s}(A, B)=\sqrt{\sum_{i=1}^{n}\left(\left(\mu_{A}\left(x_{i}\right)-\mu_{B}\left(x_{i}\right)\right)^{2}+\left(v_{A}\left(x_{i}\right)-v_{B}\left(x_{i}\right)\right)^{2}+\left(\gamma_{A}\left(x_{i}\right)-\gamma_{B}\left(x_{i}\right)\right)^{2}+\left(\pi_{A}\left(x_{i}\right)-\pi_{B}\left(x_{i}\right)\right)^{2}\right)}
$$

we have

$\left(\pi_{A}\left(x_{i}\right)-\pi_{B}\left(x_{i}\right)\right)^{2}=\left(-\mu_{A}\left(x_{i}\right)-v_{A}\left(x_{i}\right)-\gamma_{A}\left(x_{i}\right)+\mu_{B}\left(x_{i}\right)+v_{B}\left(x_{i}\right)+\gamma_{B}\left(x_{i}\right)\right)^{2}=$

$\left(\mu_{B}\left(x_{i}\right)-\mu_{A}\left(x_{i}\right)\right)^{2}+\left(v_{A}\left(x_{i}\right)-v_{B}\left(x_{i}\right)\right)^{2}+\left(\gamma_{A}\left(x_{i}\right)-\gamma_{B}\left(x_{i}\right)\right)^{2}$

$+2\left(\mu_{B}\left(x_{i}\right)-\mu_{A}\left(x_{i}\right)\left(v_{A}\left(x_{i}\right)-v_{B}\left(x_{i}\right)\right)\left(\gamma_{B}\left(x_{i}\right)-\gamma_{A}\left(x_{i}\right)\right)\right.$

v) The

normalized

Hamming

distance

is

equal

$N H_{N s}(A, B)=\frac{1}{2 n} \sum_{i=1}^{n}\left(\left|\mu_{A}\left(x_{i}\right)-\mu_{B}\left(x_{i}\right)\right|+\left|v_{A}\left(x_{i}\right)-v_{B}\left(x_{i}\right)\right|+\left|\gamma_{A}\left(x_{i}\right)-\gamma_{B}\left(x_{i}\right)\right|+\left|\pi_{A}\left(x_{i}\right)-\pi_{B}\left(x_{1}\right)\right|\right)$ 
vi) The $\quad$ normalized
$N E_{N s}(A, B)=\sqrt{\frac{1}{2 n} \sum_{i=1}^{n}\left(\left(\mu_{A}\left(x_{i}\right)-\mu_{B}\left(x_{i}\right)\right)^{2}+\left(v_{A}\left(x_{i}\right)-v_{B}\left(x_{i}\right)\right)^{2}+\left(\gamma_{A}\left(x_{i}\right)-\gamma_{B}\left(x_{i}\right)\right)^{2}+\left(\pi_{A}\left(x_{i}\right)-\pi_{B}\left(x_{i}\right)\right)^{2}\right)}$

\section{Remark 3.3}

It is easy to notice that for formulas i), ii), iii) and iv) the following is valid:
a) $0 \leq d_{N S}(A, B) \leq 2 n$
b) $0 \leq N H_{N s}(A, B) \leq 2$
c) $0 \leq e_{N s}(A, B) \leq \sqrt{2 n}$
d) $0 \leq N E_{N s}(A, B) \leq \sqrt{2}$.

\section{QUERYING VIA NEUTROSOPHIC SETS}

A query may be treated as a set of searching criteria conceived by a user. A typical query expressed in SQL is written in a following form

SELECT < list of attributes >

FROM < list of tables >

WHERE < condition >.

Its role is to select records (rows) that satisfy given condition. Each record from the table either satisfies or does not satisfy the condition and as a result we obtain a crisp set of database records that come up to query. However, as it was mentioned above, traditional query syntax requires very rigid formulation of the constraints, while for a human being a common language is a natural medium to form and express his thoughts. Now we will try to construct a query that enables a direct use of linguistic terms modeled by neutrosophic sets, i.e. a query with a following syntax:

SELECT < list of attributes >

FROM < list of tables >

WHERE <neutrosophic condition >.

Let us consider a crisp relational database with a set of attributes $\Delta=\left\{A_{1}, A_{2}, \ldots A_{n}\right\}$ and a set of records $R=\left\{r_{1}, r_{2}, \ldots r_{m}\right\}$. Let $X_{j}$ denote the universe of discourse for the attribute $A_{j}$. Moreover, let $Z: R \rightarrow X_{1} \times X_{2} \times \ldots \times X_{n}$ denote a function that determines a vector of values of all attributes corresponding to Let us define a function $U: R \rightarrow N S(R)$ which determines a neutrosophic set $R_{i}$ for each record $r_{i}$ in a following way $R_{i}=U\left(r_{i}\right)=\left\{<A_{1}, \mu_{R_{i}}\left(A_{1}\right), v_{R_{i}}\left(A_{1}\right), \sigma_{R_{i}}\left(A_{1}\right)>, \ldots<A_{n}, \mu_{R_{i}}\left(A_{n}\right), v_{R_{i}}\left(A_{n}\right), \sigma_{R_{i}}\left(A_{n}\right)>\right\}, ;$ where $\mu_{R_{i}}\left(A_{j}\right)=$ $\mu_{A_{j}}^{T}\left(z_{i j}\right), v_{R_{i}}\left(A_{j}\right)=v_{A_{j}}^{T}\left(z_{i j}\right)$, and $\sigma_{R_{i}}\left(A_{j}\right)=\sigma_{A_{j}}^{T}\left(z_{i j}\right)$.

In other words $\quad R_{i}=\left\{<A_{1}, \mu_{A_{1}}\left(z_{i 1}\right), v_{A_{i}}\left(z_{i 1}\right), \sigma_{A_{i}}\left(z_{i 1}\right)>, \ldots<A_{n}, \mu_{A_{n i}}\left(z_{i n}\right), v_{A_{i}}\left(z_{i n}\right), \sigma_{A_{i}}\left(z_{i n}\right)>\right\}$,

It is obvious that an neutrosophic set B corresponding to the best record, i.e. the record satisfying perfectly all requirements of the query, would have a following form $B=\left\{\left\langle A_{1}, 1,0,0\right\rangle, \ldots\left\langle A_{n}, 1,0,0\right\rangle\right\}$, while a neutrosophic set $\mathrm{W}$ corresponding to the worst record, i.e. the record that does not satisfy any requirements of the query, would look like : $W=\left\{\left\langle A_{1}, 0,1,1\right\rangle, \ldots\left\langle A_{n}, 0,1,1\right\rangle\right\}, \mathrm{We} \quad$ will apply neutrosophic sets $\mathrm{B}$ and $\mathrm{W}$ in our method of calculating matching degrees. They would simply constitute the upper of the attribute $A_{j}$ for the record $r_{i}$. To construct a NSquery, a suitable neutrosophic set must be defined for each attribute used in WHERE clause. Thus, actually, our NSquery is an operator T which transforms each attribute $A_{j}$ to the corresponding neutrosophic set $A_{j}{ }^{T}$ $A_{j}^{T}=\left\{<\mu_{j}^{T}(x), v_{j}^{T}(x), \sigma_{j}^{T}(x)>: x \in X_{j}\right\}$ where $\quad \mu_{j}{ }^{T}, v_{j}{ }^{T}, \sigma_{j}{ }^{T}: X_{j} \rightarrow[0,1]$ are the membership, indeterminacy and non-membership function of the defined by the neutrosophic term $\mathrm{T}$ for the attribute $A_{j}$, respectively.

As soon as we accept vague terms in queries we also have to modify our meaning of matching between the query and a record of database. It would be unreasonable to require the answer for a NS-query to be completely precise, adhering to the classical yes-no or non logic.

Now we expect the system to produce a list of records matching a query to a degree higher than a specified threes hold and to list the records according to the linear semi ordering. However, in our approach utilizing neutrosophic sets we do not have such natural linear ordering, because we have to look on three functions $\mu_{j}^{T}, v_{j}{ }^{T}, \sigma_{j}{ }^{T}$. Therefore, we will construct a desired semi ordering using distances mentioned in Sec.3. each record, i.e. $Z\left(r_{i}\right)=\left[z_{i 1}, \ldots, z_{i n}\right]$ where $z_{i j}$ is a value horizon and the lower horizon, respectively. Hence $d\left(R_{i}, B\right)$ and $d\left(R_{i}, W\right)$ denote the Hamming or

Euclidean distance of the neutrosophic set $R_{i}$ from the upper and lower horizon, respectively. These two numbers show how close is the record $r_{i}$ to the best and to the worst possible record, respectively. Of course, while querying database we are looking for records with possibly low $d(., B)$ and possibly high $d(., W)$ Therefore, let us define 
$S_{i}=1-d\left(R_{i}, B\right), \bar{S}_{i}=d\left(R_{i}, W\right)$. It is clear that a desired record should have both values $S_{i}$ and $\bar{S}_{i}$ as high as possible. An easy computation shows that for the Hamming distance we obtain:

$$
\begin{aligned}
& S_{i}=1-d_{N s}\left(R_{i}, B\right)=1-\sum_{i=1}^{n}\left(\left|\mu_{R_{i}}\left(x_{i}\right)-\mu_{B}\left(x_{i}\right)\right|+\left|v_{R_{i}}\left(x_{i}\right)-v_{B}\left(x_{i}\right)\right|+\left|\gamma_{R_{i}}\left(x_{i}\right)-\gamma_{B}\left(x_{i}\right)\right|\right) \\
& \bar{S}_{i}=d\left(R_{i}, W\right)=\sum_{i=1}^{n}\left(\left|\mu_{R_{i}}\left(x_{i}\right)-\mu_{W}\left(x_{i}\right)\right|+\left|v_{R_{i}}\left(x_{i}\right)-v_{W}\left(x_{i}\right)\right|+\left|\gamma_{R_{i}}\left(x_{i}\right)-\gamma_{W}\left(x_{i}\right)\right|\right)
\end{aligned}
$$

Similarly, we can consider the Euclidean distances $e_{N s}\left(R_{i}, B\right)$ and $e_{N s}\left(R_{i}, W\right)$ and corresponding values

$$
\begin{aligned}
& S_{i}=1-e_{N s}\left(R_{i}, B\right)=1-\sqrt{\sum_{i=1}^{n}\left(\left(\mu_{R_{i}}\left(x_{i}\right)-\mu_{B}\left(x_{i}\right)\right)^{2}+\left(v_{R_{i}}\left(x_{i}\right)-v_{B}\left(x_{i}\right)\right)^{2}+\left(\gamma_{R_{i}}\left(x_{i}\right)-\gamma_{B}\left(x_{i}\right)\right)^{2}\right)} \\
& \bar{S}_{i}=e_{N S}\left(R_{i}, W\right)=\sqrt{\sum_{i=1}^{n}\left(\left(\mu_{R_{i}}\left(x_{i}\right)-\mu_{W}\left(x_{i}\right)\right)^{2}+\left(v_{R_{i}}\left(x_{i}\right)-v_{W}\left(x_{i}\right)\right)^{2}+\left(\gamma_{R_{i}}\left(x_{i}\right)-\gamma_{W}\left(x_{i}\right)\right)^{2}\right)}
\end{aligned}
$$

Now the question is how to apply (1), (2), (3) and (4) in matching degrees computation. We suggest here three basic methods for determining matching degrees. Namely, we can calculate the matching degree for the ${ }^{\mathrm{i}}$ th record either as an

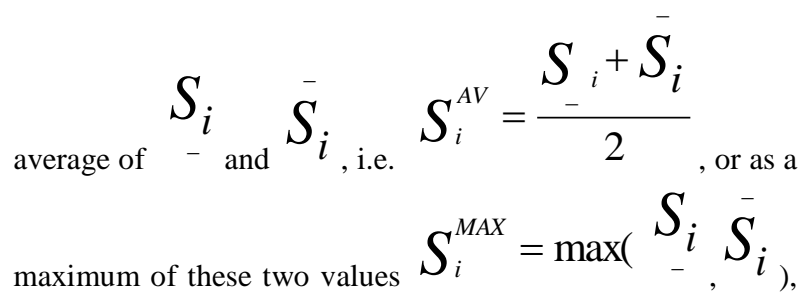
or as the minimum $S_{i}^{M I N}=\min \left(S_{-}, \bar{S}_{i}\right.$ ) It is easily seen that ${ }^{S_{i}} \leq \bar{S}_{i}$ Thus we get $S_{i}^{M A X}=S_{i}^{M I N}$. Hence using $S_{i}^{M I N}$ we restrict our consideration to the distance from the record which fits best, while using $S_{i}^{M I N}$ we consider the distance from the worst possibility only. Thus $S_{i}^{M I N}$ gives us an optimistic matching degree, $S_{i}^{M A X}$ a pessimistic one and $S_{i}^{A V}$ is a balanced one. We can also consider a natural family of operators for matching degree computation. Suppose $q \in[0,1]$ is a constant that characterizes the subjective weight attributed to the distance from the upper and the lower horizon. Then, for given q, let us define the matching degree for the record $\mathrm{i}$-th as follows $S_{i}^{q}=q S_{i}(1-q) \bar{S}_{i}$ operators discussed above are particular members of the family $\left\{S_{i}^{q}: q \in[0,1]\right\} . \quad$ Namely, $S_{i}^{A V}=S_{i}^{0.5}$, $S_{i}^{M I N}=S_{i}^{1}$ and $S_{i}^{M A X}=S_{i}^{0}$. Whatever method for calculating matching degrees (note it briefly as $S_{i}$ ) we choose, this method induces a semi ordering on a set of records. Hence we may say that a record $r_{i}$ precedes record $r_{j}$ (or is - in some sense - better) if and only if the matching degree $S_{i}$ is not smaller than $S_{j}$, i.e. $r_{i}>r_{j} \Leftrightarrow S_{i} \geq S_{j}$

Of course, this semi ordering strongly depends on the method used for calculating matching degree. We expect the system to reject the records with matching degree lower than a specified threshold. Therefore we reject the i-th record if $S_{i} \leq \xi$, where $\xi$ is a fixed number from the interval [0;1]. Hence we obtain a following algorithm of querying via neutrosophic values:

1. Take the record from the database.

2. Calculate $S_{i}$.

3. Accept the record if $S_{i} \geq \xi_{\square(\text { where }} \xi \in[0,1]$ ), otherwise reject.

4. If there are more records go to Step 1, otherwise go to Step 5 .

5. List all accepted records from the 'best' to the 'worst' according to $r_{i}>r_{j} \Leftrightarrow S_{i} \geq S_{j}$

\section{CONCLUSION}

In the present paper we have shown how to enrich fuzzy querying by the use of neutrosophic values. Since a condition in the clause WHERE may involve not only imprecise values but also such linguistic terms as fuzzy relations, and linguistic quantifiers, some other generalizations seem natural. In further work we would try to apply neutrosophic sets for modeling relations and in defining quantifiers too. However, we believe that even limited, our method enables the user to 
construct queries in a more flexible way. Some of the properties of the neutrosophic sets, Distance measures and Hesitancy Degree, These measures can be used effectively in image processing and pattern recognition. The future work will cover the application of these measures.

\section{ACKNOWLEDGMENTS}

I gratefully acknowledge the support and generosity of Benha university, faculty of computers and informatics http://www.bu.edu.eg/

\section{REFERENCES}

[1] K. Atanassov (1986), Intuitionistic fuzzy sets, Fuzzy Sets and Systems 20, 87-96

[2] K. Atanassov (1999), Intuitionistic Fuzzy Sets:Theory and Applications, Physica -Verlag.

[3] K. Atanassov, Review and new result on intuitionistic fuzzy sets, preprint IM-MFAIS-1-88, Sofia, 1988.

[4] S. A. Alblowi, A. A. Salama and Mohmed Eisa, (2014), New Concepts of Neutrosophic Sets, International Journal of Mathematics and Computer Applications Research (IJMCAR), Vol. 4, Issue 1, 59-66, 2014.

[5] P. Bosc, J. Kacprzyk, Eds. (1995), Fuzziness in Database Management Systems, Physica-Verlag, Heidelberg.

[6] P. Grzegorzewski (1998), Metrics and orders in space of fuzzy numbers, Fuzzy Sets and Systems 97, 83-94.

[7] P. Grzegorzewski (2002), Distances between intuitionistic fuzzy sets based on the Hausdorff metric, submitted to Fuzzy Sets and Systems.

[8] P. Grzegorzewski, E. Mr'owka (2002), Soft querying via intuitionistic fuzzy sets, Proceedings of the 9th International conference on Information Processing and management of Uncertainty in Knowledge-Based Systems IMPU'2002, Annecy, July 1-5, 2002, pp. 1845 1849.

[9] J. Kacprzyk, S. Zadro zny (2000), On combining intelligent querying and data mining usingfuzzy logic concepts, In: Bordogna G., Pasi G. (Eds.): Recent Issues on Fuzzy Databases, Physica-Verlag, Heidelberg, pp. $67-81$.

[10] I. M. Hanafy, A.A. Salama and K. Mahfouz, (2013),"Correlation Coefficient of Neutrosophic Sets by Centroid Method" International Journal of Probability and Statistics, 2(1), pp 9-12.

[11] I. M. Hanafy, A. A. Salama and K. Mahfouz (2012), Correlation of neutrosophic Data, International Refereed Journal of Engineering and Science (IRJES), Vol.(1), Issue 2, PP.39-43.

[12] A. A. Salama and S.A. Alblowi, (2012)," Neutrosophic Set and Neutrosophic Topological Spaces" ISOR J. mathematics (IOSR-JM), Vol. (3). Issue (4), (Sep-Oct. 2012). pp 31-35.

[13] A.A. Salama and S.A. Alblowi, (2012),"Generalized Neutrosophic Set and Generalized Neutrousophic Topological Spaces"Journal Computer Science and Engineering,Vol. (2) No. (7).pp98-102.

[14] A. A. Salama, and H. Elagamy," Neutrosophic Filters" International Journal of Computer Science Engineering and Information Technology Reseearch (IJCSEITR), Vol.3, Issue (1), Mar 2013, pp 307-312, 2013.

[15]F.Smarandach (2003)," Neutrosophic set a generalization of the intuitinistic fuzzy sets" , Proceedings of the third conference of the European Society for fuzzy logic and Technolgye, EUSFLAT, Septamper Zittau Geamany Univ. of Applied Sciences at Zittau Goerlit 2, 141-146.

[16]F.Smarandach (2013), INTRODUCTION TONEUTROSOPHIC MEASURE,NEUTROSOPHIC INTEGRAL,AND NEUTROSOPHIC PROBABILITY http://fs.gallup.unm.edu/eBooks-otherformats.htm EAN: 9781599732534

[17] EulaliaSzmidt and Janus Koeprzyk (2000), Distance between intuitionistic fuzzy sets, Fuzzy Sets and Systems 114(2000) 505-518.

[18] A. A. Salama,"Neutrosophic Crisp Points \&Neutrosophic Crisp Ideals", Neutrosophic Sets and Systems, Vol.1, No. $1,(2013)$ pp 50-54

[19] A. A. Salama and F. Smarandache, "Filters via Neutrosophic Crisp Sets", Neutrosophic Sets and Systems, Vol.1, No. 1,(2013) pp34-38

[20] .A.A. Salama," The Concept of Neutrosophic Set and Basic Properties of Neutrosophic Set Operations" WASET 2012 PARIS, FRANC, International University of Science, Engineering and Technology, 2012

[21] A. A. Salama, F. Smarandache and ValeriKroumov " Neutrosophic Crisp Sets \&Neutrosophic Crisp Topological Spaces", Neutrosophic Sets and Systems Vol.(2) pp25-30, 2014

[22] A. A. Salama, F. Smarandache and S. A. Alblowi " The Characteristic Function of a neutrosophic Set " Neutrosophic Sets and System, Accepted (2014) .

[23] A. A. Salama, Mohamed Eisa and M. M Abdelmoghny, "Neutrosophic Relations Database" International Journal of Information Science and Intelligent System, 3(1) (2014).

[24] Supriya Kumar De Ranjit Biswas and AkhilRanjan Roy, Intuiistiontic Fuzzy Database Second Int. Conf. on IFS Sofia, 3-4 Oct. 1998 NIFS 42, 34-41, 1998.

[25] L.A. Zadeh, Fuzzy Sets, Inform and Control 8338-353, 1965 